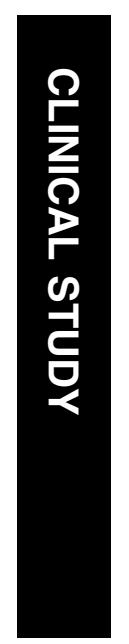

\title{
The incidence of asystole in patients undergoing strabismus surgery
}

\begin{abstract}
Purpose To investigate the incidence of asystole during strabismus surgery. Design Retrospective, noncomparative interventional case series.

Methods Six months to 80 years of age of 3628 consecutive patients who underwent strabismus surgery from October 1994 to May 2007 were enrolled.
\end{abstract}

Results Four patients $\mathbf{( 0 . 1 1 \% )}$ under general anaesthesia showed asystole during strabismus surgery. All four were adults of 28,

${ }^{1}$ Department of Anesthesiology, Seoul National University College of Medicine, SeoulMetropolitan Boramae Hospital, Seoul, Korea

\section{${ }^{2}$ Department of}

Ophthalmology, Seoul National University College of Medicine, Seoul National University Bundang

Hospital, Seongnam, Korea

Correspondence:

J-M Hwang,

Department of

Ophthalmology, Seoul National University College of Medicine, Seoul National University Bundang Hospital, 166 Gumiro, Bundang-gu, Seongnam, Gyeonggi-do 463-707, Korea

Tel: + 82-31-787-7372;

Fax: + 82-31-787-4057

E-mail: hjm@

snu.ac.kr

Received: 18 July 2007 Accepted in revised form: 31 March 2008 Published online: 16 May 2008 hypertension and three had preoperative electrocardiographic abnormalities. Three patients had previously undergone uneventful strabismus surgery.

Conclusions Asystole is likely to be encountered during strabismus surgery with an incidence of $\boldsymbol{c a} . \mathbf{0 . 1 1 \%}$.

Eye (2009) 23, 864-866; doi:10.1038/eye.2008.127; published online 16 May 2008

Keywords: asystole; strabismus surgery; oculocardiac reflex

\section{Introduction}

Patients who undergo strabismus surgery are at risk of intraoperative bradycardia due to oculocardiac reflex (OCR). OCR is defined as a reduction in heart rate (HR) of more than $20 \%$ of baseline value, dysrhythmia, or sinoatrial arrest. ${ }^{1}$ In two large series of patients that underwent strabismus surgery, the intraoperative incidences of OCR during traction on extraocular muscles was found to be 56 and $68 \% .^{2,3}$ However, no study has been undertaken to investigate the incidence of asystole during traction on extraocular muscles. Moreover, only one study has evaluated the effect of the topical application of lignocaine on 32,50 , and 53 years of age. Two patients had
S-W Min ${ }^{1}$ and J-M Hwang ${ }^{2}$

OCR. This study found a $10 \%$ frequency of cardiac arrest lasting more than $10 \mathrm{~s}$ after medial rectus stimulation. ${ }^{4}$ Another study, albeit not involving strabismus surgery, found a frequency of cardiac arrest under general anaesthesia during ophthalmological operations of $0.24 \%(2 / 822) .{ }^{5}$ Moreover, in a meta-analysis of OCR for all kinds of operations under propofol anaesthesia, one $(0.15 \%)$ in 660 cases developed asystole. ${ }^{6}$ The present study was undertaken to investigate the incidence of asystole in patients undergoing strabismus surgery under general anaesthesia.

\section{Materials and methods}

Six months to 80 years of age of 3628 consecutive patients (Table 1) who underwent strabismus surgeries under general anaesthesia by one surgeon (JMH) from October 1994 to May 2007 were included in this study.

Patients who showed any abnormal preoperative electrocardiogram (ECG) finding or any significant past or present medical disease were informed of the risks of the procedure during consultation. ECG, peripheral oxygen saturation, end tidal $\mathrm{CO}_{2}\left(\mathrm{ETCO}_{2}\right)$, and noninvasive blood pressure (BP) were monitored in all patients. General anaesthesia was induced with intravenous thiopental sodium $(5 \mathrm{mg} / \mathrm{kg})$, oxygen (100\%)-enflurane or sevoflurane, and vecuronium bromide $(0.12 \mathrm{mg} / \mathrm{kg})$. Concentrations of enflurane and sevoflurane were adjusted according to anaesthetic depth which was maintained and adjusted according to the BP and HR being stable and 10-20\% lower than those in awake, which is generally accepted anaesthetic maintenance for its convenience and simplicity. $\mathrm{ETCO}_{2}$ was maintained within the normal range (30-40 mmHg). After 3-5 min of mask ventilation, patients were intubated and anaesthesia was maintained with nitrous oxide 
(50\%), oxygen $(50 \%)$, and enflurane or sevoflurane. Patients were not given any anticholinergics before or at the beginning of the procedures, however, a syringe filled with atropine was always kept bedside ready for the urgent case of bradycardia.

Data including age, gender, operative eye, extraocular muscle, surgical procedure, past medical history, anaesthetic technique, and the preoperative ECG findings of patients who showed asystole during extraocular muscle traction, were collected.

\section{Results}

Four of the 3628 patients $(0.11 \%)$ who underwent strabismus surgery under general anaesthesia showed asystole lasting more than $6 \mathrm{~s}$ after right inferior rectus, lateral rectus, medial rectus, or left lateral rectus stimulation, respectively, during strabismus surgery. All of four were adults of 28, 32, 50, and 53 years of age. In terms of medical history, two had hypertension and were controlled at the time of surgery; three patients showed preoperative ECG abnormalities of first-degree atrioventricular block, supraventricular premature beats, and premature ventricular contractions, respectively (Table 2). Only one of the patients with hypertension was managed with atenolol $25 \mathrm{mg}$ and terazocin $1 \mathrm{mg}$ p.o. q.d.

Two patients had undergone one previous uneventful strabismus procedure, namely, left inferior myectomy in one and recessions of the right superior and medial recti in the other. One patient had undergone two uneventful strabismus surgeries, that is, unilateral lateral rectus

Table 1 Age distribution of 3628 patients

\begin{tabular}{lc}
\hline Age (years) & No. of patients $(\%)$ \\
\hline $0-10$ & $2676(73.8)$ \\
$11-20$ & $357(9.9)$ \\
$21-30$ & $242(6.7)$ \\
$31-40$ & $150(4.1)$ \\
$31-40$ & $102(2.8)$ \\
$41-50$ & $59(1.6)$ \\
$51-60$ & $30(0.8)$ \\
$61-70$ & $12(0.3)$ \\
Total & $3628(100.0)$ \\
\hline
\end{tabular}

recession and medial rectus resection. However, their surgeries were not so difficult in spite of redoing a surgery in the same eye.

In these four patients that developed asystole, anaesthesia was maintained with nitrous oxide (50\%), oxygen (50\%), and enflurane. QRS signal on the ECG disappeared and no pulse was palpated immediately after traction was applied. After traction was released, HRs recovered to the level before hooking the muscle in all four patients. Atropine sulphate $0.5 \mathrm{mg}$ was given intravenously to all patients to proceed with surgery. HR was then maintained at or above the level observed before muscle hooking. In all four, surgery proceeded without further problems. The extraocular muscles, which on traction resulted in asystole, were the right inferior rectus, lateral rectus, medial rectus, and left lateral rectus. None of the patients suffered apparent sequelae due to the episode of asystole and all were discharged later during the day of surgery.

\section{Discussion}

Asystole or anaesthetic death associated with OCR during strabismus surgery have been reported. ${ }^{5-10}$ However, all published studies are case reports, and thus, cannot provide statistical data. Here, we present the frequency of asystole in patients who underwent strabismus surgery under general anaesthesia among a large number of surgical cases.

In 1972, Smith et $a l^{8}$ reported two cases of sinus arrest after traction of the extraocular muscles, despite apparent adequate protection with retrobulbar block and intravenous atropine. Moreover, sinus rhythms returned spontaneously after releasing the muscle from traction. Gold et $a l^{9}$ also reported two cases of asystole during strabismus surgery.

In this study, all four patients who developed asystole were adults. Two patients had hypertension, and three had preoperative ECG abnormalities. Thus, it might be concluded that adults with a cardiovascular problem, such as hypertension or a preoperative ECG abnormality, are at higher risk of asystole during strabismus surgery. However, two patients in another report ${ }^{5}$ were children

Table 2 Demographic characteristics of the four patients who developed asystole during strabismus surgery

\begin{tabular}{llllll}
\hline & Sex/age (years) & PMHx & Preoperative ECG & Previous ocular surgeries & Muscles on traction \\
\hline 1 & $\mathrm{M} / 28$ & None & Normal & LIO myectomy & RIR \\
2 & $\mathrm{M} / 32$ & None & First degree AV block & None & RLR \\
3 & $\mathrm{M} / 53$ & HT & SVPB & Repairs of blowout fractures, RSR and MR rec & LLR \\
4 & F/50 & HT & PVC & B) LR rec \& MR res & RMR \\
\hline
\end{tabular}

PMHx, past medical history; HT, hypertension; ECG, electrocardiogram; AV, atrioventricular; SVPB, supraventricular premature beats; PVC premature ventricular contractions; R, right; L, left; IO, inferior oblique; SR, superior rectus; MR, medial rectus; LR, lateral rectus; rec, recession; res, resection. 
of 4 and 13 years of age, and had no known preoperative medical condition.

Interestingly in this study, three of the four patients had undergone one or two uneventful strabismus surgeries. Therefore, a previous uneventful surgery did not guarantee subsequent uneventful surgery. No report of increased OCR risk due to repeat operation has been previously issued.

All four patients who developed asystole were anaesthetized using enflurane. Although studies have compared OCR frequencies for propofol and inhalational anaesthetics, such as halothane,$^{13}$ isoflurane, ${ }^{14,15}$ or sevoflurane, ${ }^{16,17}$ and between inhalational anaesthetics such as sevoflurane and halothane, ${ }^{18}$ no study has compared the frequency of OCR for enflurane and sevoflurane. Comparisons between enflurane and other anaesthetics are thus required in this context. Rapid acting narcotics such as remifentanil, sufentanil, or fentanyl were reported to enhance the degree of bradycardia due to the OCR in the absence of anticholinergic blockade. ${ }^{19}$ In none of the four patients in this study, narcotics were used intraas well as perioperatively.

Intraoperative cardiac asystole during strabismus surgery causes ophthalmologists and anaesthesiologists considerable concern. Preventive measures, such as the administration of an anticholinergic drug to abolish OCR, have been investigated, but results are controversial because of the introduction of potential risks of arrhythmia and tachycardia. ${ }^{11,12}$ In this study, all four patients showed HR recovery as soon as traction on the extraocular muscle was relieved, and surgery was continued in all cases using intravenous injection of atropine sulphate; thus an awareness of the possibility of OCR may be sufficient to manage asystole should it occur.

This study has important limitations stemming from its retrospective design and the involved anaesthesiologists. However, a happening of asystole was too fearful to forget and was kept recorded, therefore the incidence may be reliable. However, a prospective study could be helpful to determine the incidence of asystole during strabismus surgeries. All of anaesthesiologists were trained in the same department of one university, 'Department of Anaesthesiology of Seoul National University' and adopted the same protocol for the general anaesthesia. Therefore, even though many anaesthesiologists were involved, the methods were quite same.

This study shows that asystole is likely to be encountered in an adult of 28-53 years of age during strabismus surgery with at a frequency of $0.11 \%$. It is important that the possibility of asystole is recognized by anaesthesiologists and ophthalmologists.

The authors declare that they have no vested interest that could be construed to have inappropriately influenced this study.

\section{References}

1 Loewinger J, Friedmann-Neiger I, Cohen M, Levi E. Effects of atracurium and pancuronium on the oculocardiac reflex in children. Anesth Analg 1991; 73: 25-28.

2 Welhaf WR, Johnson DC. The oculocardiac reflex during extraocular muscle surgery. Arch Ophthalmol 1965; 73: 43-48.

3 Apt L, Isenberg S, Gaffney WL. The oculocardiac reflex in strabismus surgery. Am J Ophthalmol 1973; 76: 533-536.

4 Ruta U, Möllhoff T, Markodimitrakis H, Brodner G. Attenuation of the oculocardiac reflex after topically applied lignocaine during surgery for strabismus in children. Eur J Anaesthesiol 1996; 13: 11-15.

5 Galkowska I, Nizieñska E. Cardiac arrest during ophthalmological operations. Anaesth Resusc Intensive Ther 1975; 3: 197-200.

6 Tramèr MR, Moore RA, McQuay HJ. Propofol and bradycardia: causation, frequency and severity. $\mathrm{Br} J$ Anaesth 1997; 78: 642-651.

7 Sorenson EJ, Gilmore JE. Cardiac arrest during strabismus surgery; a preliminary report. Am J Ophthalmol 1956; 41: 748-752.

8 Smith RB, Douglas H, Petruscak J. The oculocardiac reflex and sino-atrial arrest. Can Anaesth Soc J 1972; 19 138-142.

9 Gold RS, Pollard Z, Buchwald IP. Asystole due to the oculocardiac reflex during strabismus surgery: a report of two cases. Ann Ophthalmol 1988; 20: 473-477.

10 Cooper J, Medow N, Dibble C. Mortality rate in strabismus surgery. J Am Optom Assoc 1982; 53: 391-395.

11 Jedeikin RJ, Hoffman S. The oculocardiac reflex in eye-surgery anesthesia. Anesth Analg 1977; 56: 333-334.

12 Batterbury M, Wong D, Williams R, Kelly J, Mostafa SM. Peribulbar anaesthesia: failure to abolish the oculocardiac reflex. Eye 1992; 6: 293-295.

13 Watcha MF, Simeon RM, White PF, Stevens JL. Effect of propofol on the incidence of postoperative vomiting after strabismus surgery in pediatric outpatients. Anesthesiology 1991; 75: 204-209.

14 Snellen FT, Vanacker B, Van Aken H. Propofol-nitrous oxide versus thiopental sodium-isoflurane-nitrous oxide for strabismus surgery in children. J Clin Anesth 1993; 5: 37-41.

15 Tramer MR, Sansonetti A, Fuchs-Buder T, Rifat K. Oculocardiac reflex and postoperative vomiting in paediatric strabismus surgery. A randomized controlled trial comparing four anaesthetic techniques. Acta Anaesthesiol Scand 1998; 42: 117-123.

16 Hahnenkamp K, Honemann CW, Fischer LG, Durieux ME, Muehlendyck $\mathrm{H}$, Braun U. Effect of different anaesthetic regimes on the oculocardiac reflex during paediatric strabismus surgery. Paediatr Anaesth 2000; 10: 601-608.

17 Gurkan Y, Kilickan L, Toker K. Propofol-nitrous oxide versus sevoflurane-nitrous oxide for strabismus surgery in children. Paediatr Anaesth 1999; 9: 495-499.

18 Allison CE, De Lange JJ, Koole FD, Zuurmond WW, Ros $\mathrm{HH}$, van Schagen NT. A comparison of the incidence of the oculocardiac and oculorespiratory reflexes during sevoflurane or halothane anesthesia for strabismus surgery in children. Anesth Analg 2000; 90: 306-310.

19 Arnold RW, Jensen PA, Kovtoun TA, Maurer SA, Schultz JA. The profound augmentation of the oculocardiac reflex by fast acting opioids. Binocul Vis Strabismus Q 2004; 19: 215-222. 